Electronic supplementary material available online for this article.

\title{
Sigma metrics for assessing the analytical quality of clinical chemistry assays: a comparison of two approaches
}

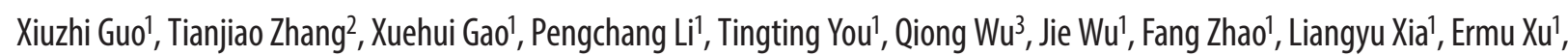
Ling Qiu', Xinqi Cheng ${ }^{* 1}$

\footnotetext{
${ }^{1}$ Department of Laboratory Medicine, Peking Union Medical College Hospital, Chinese Academic Medical Science and Peking Union Medical College, Beijing, P.R. China

${ }^{2}$ National Center for Clinical Laboratories, Beijing Hospital, National Center of Gerontology, Beijing, P.R. China

${ }^{3}$ Clinical Laboratory, Affiliated Hospital of Chifeng University, Inner Mongolia, P.R. China

The first two authors contributed equally to this work.
}

*Corresponding author: chengxq@pumch.cn

\begin{abstract}
Introduction: Two approaches were compared for the calculation of coefficient of variation (CV) and bias, and their effect on sigma calculation, when different allowable total error (TEa) values were used to determine the optimal method for Six Sigma quality management in the clinical laboratory.

Materials and methods: Sigma metrics for routine clinical chemistry tests using three systems (Beckman AU5800, Roche C8000, Siemens Dimension) were determined in June 2017 in the laboratory of Peking Union Medical College Hospital. Imprecision (CV\%) and bias (bias\%) were calculated for ten routine clinical chemistry tests using a proficiency testing (PT)- or an internal quality control (IQC)-based approach. Allowable total error from the Clinical Laboratory Improvement Amendments of 1988 and the Chinese Ministry of Health Clinical Laboratory Center Industry Standard (WS/ T403-2012) were used with the formula: Sigma $=(\mathrm{TEa}-$ bias $) /\left(\mathrm{V}\right.$ to calculate the Sigma metrics $\left(\sigma_{\mathrm{CLIA}} \sigma_{\mathrm{WS} / \mathrm{T}}\right)$ for each assay for comparative analysis. Results: For the PT-based approach, eight assays on the Beckman AU5800 system, seven assays on the Roche 8000 system and six assays on the Siemens Dimension system showed $\sigma_{\text {CLIA }}>3$. For the IQC-based approach, ten, nine and seven assays, respectively, showed $\sigma_{\text {CLIA }}>3$. Some differences in $\sigma$ were therefore observed between the two calculation methods and the different TEa values.

Conclusions: Both methods of calculating $\sigma$ can be used for Six Sigma quality management. In practice, laboratories should evaluate Sigma multiple times when optimizing a quality control schedule.
\end{abstract}

Key words: Six Sigma; proficiency testing; internal quality control; allowable total error

\section{Introduction}

Clinical laboratory testing results are important for ensuring patient safety. Approximately two-thirds of important clinical decisions on patient management are based on laboratory test results (1). Therefore, continuous improvement and the minimizing of errors in testing are the major goals of every clinical laboratory. Six Sigma $(6 \sigma)$ quality management is a data-based, customer-centered, advanced quality management model that has been recently developed and is used globally. Following its introduction in clinical laboratories, 60 quality management has become a major research focus (2). Sigma ( $\sigma$ ) metrics evaluate process capability. The clinical application of $6 \sigma$ quality management involves the combined use of quality requirements and laboratory performance to quantitatively evaluate whether a laboratory meets clinical testing standards. This evaluation is typi- 
cally based on the expected defect rate. The ultimate goal of $6 \sigma$ quality management is to implement laboratory risk management and thus ensure patient safety.

Several studies on the application of $6 \sigma$ management in laboratory testing have been reported, including studies on theoretical methods and their significance, the evaluation of performance of different assays, and the optimization of quality control (QC) schedules based on performance evaluation (3-5). Sigma metrics quantitatively estimates quality based on the traditional parameters used in the clinical laboratory: allowable total error (TEa), bias and imprecision. Imprecision is usually expressed as standard deviation (SD) or coefficient of variation (CV). However, the quality requirements of various sources may be associated with differences within the same assay, which can affect the parameter selection. Furthermore, different approaches to bias and CV calculation may influence the final $\sigma$ calculation. Laboratories should thus be aware of such sources of variation when $\sigma$ management is applied, as they may modulate the value of $\sigma$ and the accuracy of $\sigma$ measurement. For example, previous studies have reported bias in external quality assessment (EQA) survey reports (e.g., College of American Pathologists or the Randox International Quality Assessment Scheme), whereas CV is derived from the cumulative coefficient of variation of internal quality control (IQC) materials (6-9). However, a strong correlation has been established between CV and the concentration of the test substance. If the concentration of IQC materials differs significantly from that of proficiency testing (PT) samples used in bias calculation, this method is inadequate for calculating $\sigma$. Bias may also be derived from reagent package inserts (10). Given that the cumulative average value of QC material may change over time, it is inappropriate to use the target value from manufacturers' QC material when calculating bias. In fact, with the continuous development of information technology, vendors can statistically analyse IQC data from the majority of laboratories to determine a more appropriate "group mean". This approach can facilitate inter-laboratory quality management based on IQC, which has become popular among clinical laboratories. Using this group mean to calculate for bias has been shown to be a convenient and reliable method.

Therefore, in the present study, we aimed to compare two approaches to the calculation of CV and bias and the effect on $\sigma$ calculation at different TEa. The two methods used to calculate the $\sigma$ value for 10 routine biochemical assays on three different analysers were a PT-based approach, where materials for routine clinical chemistry from the China National Center for Clinical Laboratories (NCCL) were evaluated for imprecision (CV\%) in each assay and bias (bias\%) was calculated by comparison with the group mean for each PT sample in the NCCL report; and an IQC-based approach, where IQC results were used to calculate the CV\% of each assay and bias\% was calculated by comparison with the global group mean. Both methods thus harmonized the source of bias and CV derived from the same sample, based on which variations in $\sigma$ were compared despite the different sources of parameters. This method of evaluating $\sigma$ has not previously been reported.

\section{Materials and methods}

\section{Materials}

Ten assays were evaluated using the manufacturer/analyser combinations routinely used in Peking Union Medical College Hospital (Table 1). All reagents and calibrators for the three analysers were obtained from the original manufacturer except for creatinine (CREA; Maccura Biotechnology Co., Ltd., Chengdu, China) and bilirubin, total (BT; Wako Pure Chemical Industries, Ltd., Osaka, Japan) on the Beckman AU5800 system (Beckman Coulter, Inc., Brea, USA).

\section{Methods}

Sample preparation. PT materials (five lots: 201721201725) for routine clinical chemistry were provided by NCCL (Peking, China). Samples were prepared using an analytical balance with an accuracy of $0.001 \mathrm{~g}$. Powdered samples were dissolved in 3 $\mathrm{mL}$ deionized water, capped, maintained at room temperature for $30 \mathrm{~min}$ and gently mixed until 
TABLE 1. Reagents, equipment and number of laboratories included in each instrument group for NCCL proficiency testing, and number of laboratories included in the Bio-Rad global report for the same instrument/method

\begin{tabular}{|c|c|c|c|c|c|c|c|c|c|c|}
\hline \multirow{2}{*}{ Analyser } & \multicolumn{10}{|c|}{ Number of laboratories in NCCL proficiency testing/Bio-Rad global report } \\
\hline & Alb & ALT & BT & Glc & CREA & Urea & K & $\mathrm{Na}$ & $\mathrm{Cl}$ & $\mathrm{Ca}$ \\
\hline $\begin{array}{l}\text { Beckman } \\
\text { AU5800* }\end{array}$ & $207 / 37$ & $221 / 148$ & $220 / 60^{\S}$ & $208 / 48$ & $138 / 22 \|$ & $210 / 22$ & $371 / 46$ & $359 / 46$ & $364 / 44$ & $202 / 39$ \\
\hline $\begin{array}{l}\text { Roche } \\
\mathrm{C}^{2} 000^{+}\end{array}$ & $419 / 26$ & $410 / 16$ & $369 / 26$ & $392 / 32$ & $384 / 22$ & $399 / 22$ & $401 / 36$ & $388 / 36$ & $387 / 35$ & $395 / 25$ \\
\hline $\begin{array}{c}\text { Siemens } \\
\text { Dimensionf }\end{array}$ & $7 / 60$ & $6 / 16$ & $6 / 24$ & $8 / 68$ & $7 / 37$ & $8 / 43$ & $8 / 57$ & $8 / 56$ & $8 / 54$ & $7 / 62$ \\
\hline
\end{tabular}

*Beckman, Beckman Coulter, Inc., Brea, CA. †Roche, Hoffmann-La Roche Ltd., Basel, Switzerland. ${ }^{\ddagger}$ Siemens, Siemens Healthcare Diagnostics, Inc., DE, US. \$Wako, Wako Pure Chemical Industries, Ltd., Osaka, Japan. \|Maccura, Maccura Biotechnology Co., Ltd., Chengdu, China. Alb - albumin. ALT - alanine aminotransferase. BT - bilirubin, total. Glc - glucose. CREA - creatinine. K - potassium. $\mathrm{Na}$ - sodium. Cl - chlorides. Ca - calcium. NCCL - China National Center for Clinical Laboratories, Peking, China.

completely dissolved. Samples were protected from light and stored between $2{ }^{\circ} \mathrm{C}$ and $8^{\circ} \mathrm{C}$ until use within 7 days.

Imprecision evaluation. During the PT period (June 5 - 9, 2017), the Clinical Laboratory Standards Institute (CLSI) EP15A3 protocol was followed, with the same sample tested five times daily for albumin (Alb), alanine aminotransferase (ALT), potassium $(\mathrm{K})$, sodium $(\mathrm{Na})$, chloride $(\mathrm{Cl})$, calcium $(\mathrm{Ca})$, total bilirubin (BT), glucose (Glc), creatinine (CREA, enzymatic), and urea (Urea) on each analyser for five consecutive days (11). The mean, SD (within laboratory) and CV (within laboratory) for each test item was calculated.

Bias calculation. Based on NCCL routine clinical chemistry requirements, the mean value of the instrument group (excluding data more than two standard deviations away from the mean) was used to verify the target value of each assay. Our mean $(\mathrm{N}=25)$ for each assay from the different analysers was calculated as described above. Bias\% was determined as (our mean - mean of all laboratories using the same instrument and method) / (mean of all laboratories using the same instrument and method) $\times 100$.

Bio-Rad (Bio-Rad Laboratories, Inc., Hercules, CA, US) Liquid assay multiqual QC materials (694/696; lot numbers 45751, 45753) were used daily to monitor internal testing quality. All assays participated in the Bio-Rad global report. Data were collected from internal QC at Peking Union Medical College
Hospital from January 1, 2017 to June 30, 2017. Monthly (June 2017) mean, SD and CV were calculated. Bias was calculated based on target value, which was averaged from the Bio-Rad global report for the same assay performed with the same instrument/method.

Sigma calculation and data analysis. According to quality requirements, the formulas $\sigma=$ (TEa |Bias\%|) / CV (for percentage) and $\sigma=($ TEa $-\mid$ Bias $\mid)$ / SD (for concentration value) were used to calculate $\sigma$ metrics for each assay. The allowable total error of each assay was based on the American Clinical Laboratory Improvement Amendments of 1988 (CLIA '88) and People's Republic of China Health Industry Standard (WS/T403-2012), designated as $\mathrm{TEa}_{\mathrm{CLIA}}$ and $\mathrm{TEa}_{\mathrm{WS} / \mathrm{T}}$, respectively $(12,13)$. The specific requirements of the 10 assays are listed in Table 2. Excel 2010 software (Microsoft Corporation, Redmond, Washington State, US) was used for data analysis and graphing.

\section{Results}

\section{PT Sigma metrics}

The $\sigma$ values calculated using two TEa sources $\left(\sigma_{\mathrm{WS} / \mathrm{T}}\right.$ and $\left.\sigma_{\mathrm{CLIA}}\right)$ for the three analysers are shown in Table 3. The $\mathrm{TEa}_{\mathrm{CLIA}}$ used absolute bias in the $\mathrm{K}$, $\mathrm{Na}$ and $\mathrm{Ca}$ assays, whereas $\mathrm{TEa}_{\mathrm{WS} / \mathrm{T}}$ used percentage bias in all other assays and was more stringent than $\mathrm{TEa}_{\mathrm{CLIA}}$. We showed that $\sigma_{\mathrm{WS} / \mathrm{T}}<\sigma_{\mathrm{CLIA}}$ for all 
TABLE 2. Allowable total error derived from the US Clinical Laboratory Improvement Amendments 1988 (CLIA ‘88) and the People's Republic of China Health Industry Standard (WS/T403-2012)

\begin{tabular}{ccc}
\hline \multirow{2}{*}{ Item } & TEa $_{\text {CLIA }}$ & TEa $_{\text {Ws/T }}$ \\
\cline { 2 - 3 } & bias\% (absolute value) & bias\% \\
\hline Alb & 10 & 6 \\
BT & 20 & 16 \\
Glc & $20(6.84)$ & 15 \\
CREA & $10(0.33)$ & 7 \\
Urea & $15(26.5)$ & 12 \\
K & $9(1)$ & 8 \\
Na & $(0.5)$ & 6 \\
Cl & $(4)$ & 4 \\
Ca & 5 & 4 \\
\hline
\end{tabular}

$\mathrm{TEa}_{\mathrm{CLIA}}$ - allowable total error derived from US Clinical Laboratory Improvement Amendments 1988 (CLIA '88). $\mathrm{TEa}_{\text {WS/T }}$ - allowable total error derived from the People's Republic of China Health Industry Standard (WS/T403-2012). Alb - albumin, g/L. ALT - alanine aminotransferase, U/L $37^{\circ} \mathrm{C}$. BT - bilirubin, total, $\mu \mathrm{mol} / \mathrm{L}$. Glc - glucose, mmol/L. CREA creatinine, $\mu \mathrm{mol} / \mathrm{L}$. Urea, $\mathrm{mmol} / \mathrm{L}$. K - potassium, $\mathrm{mmol} / \mathrm{L}$. $\mathrm{Na}$ sodium, mmol/L. Cl - chlorides, mmol/L. Ca - calcium, mmol/L. assays except $\mathrm{Na}$. For $\sigma_{\mathrm{WS} / \mathrm{T}}$, only the Siemens $\mathrm{Di}$ mension analyser achieved $\sigma \sigma$ for BT testing in all five lots. The $3 \sigma$ level was achieved for ALT, CREA and $\mathrm{K}$ on all three analysers; $\mathrm{BT}, \mathrm{Glc}$ and $\mathrm{Na}$ on the Roche $\mathrm{C} 8000$ analyser; and $\mathrm{Cl}$ and $\mathrm{Ca}$ on the Beckman AU5800 analyser.

The $\sigma_{\mathrm{CLIA}}$ calculation based on $\mathrm{TEa}_{\mathrm{CLIA}}$ showed that eight assays (all except Urea and $\mathrm{Na}$ ) achieved $\sigma>$ 3 on the Beckman AU5800 analyser. Among the eight, CREA and $\mathrm{K}$ reached $6 \sigma$ levels in the five lot numbers during proficiency testing; four assays (ALT, BT, Cl and Ca) achieved 5 0 . For the Roche C8000 analyser, all assays except Urea, $\mathrm{Na}$ and $\mathrm{Cl}$

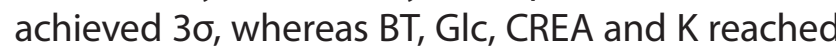
$6 \sigma$. For the Siemens Dimension analyser, BT and $\mathrm{K}$ reached $6 \sigma ; A L T$, CREA and Urea reached 40; and Alb, Glc, Na (201721, 201724, 201725) and Cl (201721) were $<3 \sigma$ at certain concentrations.

Significant differences in $\sigma_{\mathrm{CLIA}}$ values were observed using the same assay at the same concentration but for different analysers. More intuitive $\sigma_{\mathrm{CLIA}}$ levels for all assays at different concentrations

TABLE 3. Sigma metrics for the proficiency testing (PT)-based approach

\begin{tabular}{|c|c|c|c|c|c|c|c|c|c|c|c|c|c|c|c|c|}
\hline \multirow[b]{2}{*}{ Item } & \multirow[b]{2}{*}{$\begin{array}{l}\text { Sample } \\
\text { number }\end{array}$} & \multicolumn{3}{|c|}{ Mean } & \multicolumn{3}{|c|}{ CV (\%) } & \multicolumn{3}{|c|}{ Bias (\%) } & \multicolumn{3}{|c|}{$\sigma_{\mathrm{CLIA}}$} & \multicolumn{3}{|c|}{$\sigma_{\mathrm{WS} / \mathrm{T}}$} \\
\hline & & 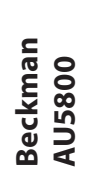 & 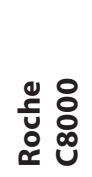 & 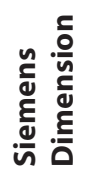 & 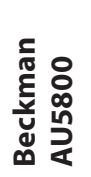 & 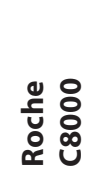 & 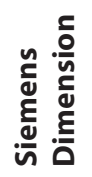 & 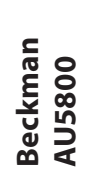 & 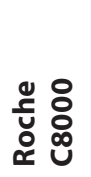 & 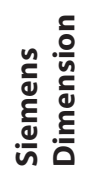 & 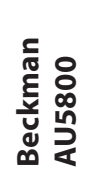 & 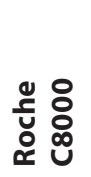 & 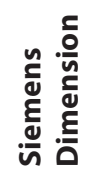 & 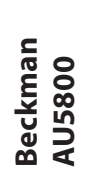 & 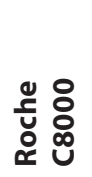 & 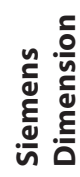 \\
\hline \multirow[t]{5}{*}{ Alb } & 201721 & 46 & 49 & 51 & 1.0 & 1.4 & 0.8 & -1.2 & -1.0 & 5.8 & 8.4 & 6.6 & 5.2 & 4.6 & 3.7 & $0.3^{+}$ \\
\hline & 201722 & 24 & 26 & 26 & 1.2 & 3.0 & 0.8 & -2.2 & 0.5 & 2.6 & 6.4 & 3.2 & 9.5 & 3.1 & $1.9^{\dagger}$ & 4.4 \\
\hline & 201723 & 32 & 35 & 35 & 1.0 & 2.1 & 1.3 & -1.8 & -0.4 & 3.6 & 8.5 & 4.5 & 4.8 & 4.4 & $2.6^{+}$ & $1.8^{\dagger}$ \\
\hline & 201724 & 46 & 49 & 52 & 2.3 & 1.6 & 1.1 & -1.1 & -1.5 & 7.2 & 3.8 & 5.5 & $2.6^{+}$ & $2.1^{\dagger}$ & $2.9^{\dagger}$ & $-1.1^{\dagger}$ \\
\hline & 201725 & 37 & 40 & 41 & 1.3 & 2.1 & 0.8 & -2.9 & -2.2 & 2.9 & 5.5 & 3.8 & 9.4 & $2.4^{\dagger}$ & $1.9^{\dagger}$ & 4.1 \\
\hline \multirow[t]{5}{*}{ ALT } & 201721 & 160 & 153 & 164 & 0.6 & 1.0 & 0.9 & -2.8 & -2.3 & -1.3 & 27.7 & 18.3 & 21.5 & 21.3 & 14.2 & 16.9 \\
\hline & 201722 & 31 & 29 & 32 & 3.0 & 4.0 & 4.6 & -2.8 & -1.1 & 0.7 & 5.8 & 4.7 & 4.2 & 4.5 & 3.7 & 3.3 \\
\hline & 201723 & 77 & 74 & 81 & 0.8 & 2.7 & 2.4 & -3.4 & -3.3 & 0.1 & 19.8 & 6.2 & 8.2 & 15 & 4.7 & 6.6 \\
\hline & 201724 & 144 & 137 & 149 & 0.8 & 1.3 & 2.0 & -2.5 & -2.6 & 0.5 & 21.1 & 13.1 & 9.6 & 16.2 & 10.1 & 7.7 \\
\hline & 201725 & 100 & 97 & 105 & 1.2 & 1.7 & 1.8 & -4.7 & -3.4 & -2.6 & 13 & 10 & 9.7 & 9.6 & 7.6 & 7.5 \\
\hline \multirow[t]{5}{*}{ BT } & 201721 & 128.1 & 121.1 & 129.4 & 1.1 & 0.8 & 0.7 & -6.3 & -4.0 & -6.8 & 13 & 19.6 & 18.6 & 8.3 & 13.5 & 11.5 \\
\hline & 201722 & 12.5 & 11.3 & 13.8 & 1.3 & 1.8 & 2.2 & -7.1 & -8.5 & 1.7 & $36.8^{*}$ & $27.6^{*}$ & $22.0^{*}$ & 6.3 & 3.6 & 6.1 \\
\hline & 201723 & 25.4 & 23.5 & 27.8 & 0.9 & 1.4 & 1.3 & -14.6 & -7.4 & 0.1 & 11.3 & 15 & 18.9 & $0.4^{\dagger}$ & 5.5 & 11.7 \\
\hline & 201724 & 85.9 & 82.0 & 89.1 & 1.0 & 1.9 & 0.6 & -4.2 & -4.9 & -5.9 & 16 & 7.8 & 22.7 & 11 & 5.2 & 14.7 \\
\hline & 201725 & 38.16 & 35.9 & 41.0 & 1.3 & 1.2 & 1.3 & -12.9 & -7.8 & -4.0 & 5.6 & 10.4 & 12.3 & $1.7^{\dagger}$ & 6.2 & 8.4 \\
\hline
\end{tabular}




\begin{tabular}{|c|c|c|c|c|c|c|c|c|c|c|c|c|c|c|c|c|}
\hline \multirow[b]{2}{*}{ Item } & \multirow[b]{2}{*}{$\begin{array}{l}\text { Sample } \\
\text { number }\end{array}$} & \multicolumn{3}{|c|}{ Mean } & \multicolumn{3}{|c|}{ CV (\%) } & \multicolumn{3}{|c|}{ Bias (\%) } & \multicolumn{3}{|c|}{$\sigma_{\text {CLIA }}$} & \multicolumn{3}{|c|}{$\sigma_{\mathrm{WS} / \mathrm{T}}$} \\
\hline & & 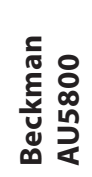 & 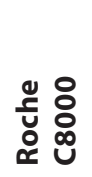 & 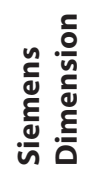 & 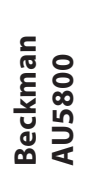 & 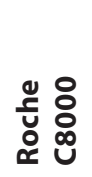 & 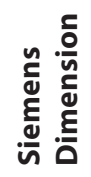 & 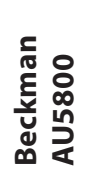 & 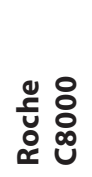 & 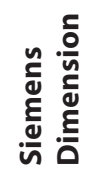 & 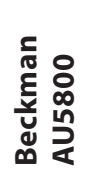 & 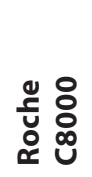 & 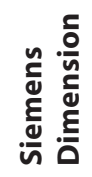 & 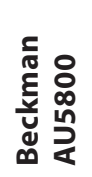 & 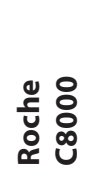 & 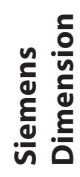 \\
\hline \multirow[t]{5}{*}{ Glc } & 201721 & 13.4 & 13.3 & 14.2 & 1.2 & 1.4 & 1.6 & -2.7 & -2.1 & 3.5 & 6.1 & 5.6 & 4.1 & 3.6 & 3.5 & $2.2^{\dagger}$ \\
\hline & 201722 & 3.8 & 3.7 & 4.0 & 1.1 & 1.1 & 2.2 & -3.0 & -2.9 & 5.5 & 6.6 & 6.7 & $2^{+}$ & 3.8 & 3.9 & $0.7^{\dagger}$ \\
\hline & 201723 & 6.6 & 6.6 & 7.1 & 1.4 & 1.1 & 2.4 & -3.1 & -2.8 & 4.5 & 5 & 6.9 & $2.3^{\dagger}$ & $2.8^{+}$ & 4 & $1.1^{+}$ \\
\hline & 201724 & 10.8 & 10.8 & 11.5 & 1.3 & 1.2 & 1.8 & -2.8 & -2.6 & 4.3 & 5.4 & 6.2 & 3.2 & 3.2 & 3.7 & $1.5^{\dagger}$ \\
\hline & 201725 & 7.3 & 7.2 & 7.8 & 1.4 & 0.9 & 1.4 & -4.5 & -4.5 & 2.4 & 3.8 & 6.5 & 5.3 & $1.7^{\dagger}$ & 3 & 3.2 \\
\hline \multirow[t]{5}{*}{ CREA } & 201721 & 466 & 464 & 452 & 0.8 & 1.2 & 0.7 & -0.2 & 2.1 & 0.4 & 19.4 & 11 & 21 & 15.5 & 8.4 & 16.7 \\
\hline & 201722 & 85 & 88 & 82 & 0.8 & 1.2 & 2.1 & 9.6 & 5.4 & 0.8 & $28.8^{*}$ & $20.2^{*}$ & $14.9^{*}$ & 3.1 & 5.3 & 5.3 \\
\hline & 201723 & 223 & 225 & 218 & 0.6 & 1.2 & 2.9 & 2.9 & 1.8 & 1.5 & 19.3 & 11.3 & 4.7 & 14.5 & 8.7 & 3.7 \\
\hline & 201724 & 469 & 467 & 455 & 0.8 & 1.1 & 1.2 & -0.7 & 1.4 & -0.7 & 17.4 & 12.2 & 11.9 & 13.7 & 9.5 & 9.4 \\
\hline & 201725 & 289 & 289 & 284 & 0.7 & 1.0 & 1.0 & -0.2 & -0.2 & -0.1 & 21.2 & 14.8 & 14.9 & 16.9 & 11.8 & 11.9 \\
\hline \multirow[t]{5}{*}{ Urea } & 201721 & 17.41 & 17.58 & 18.17 & 2.0 & 3.6 & 1.3 & -3.9 & -2.6 & 0.2 & $2.6^{\dagger}$ & $1.8^{\dagger}$ & 7.1 & $2.1^{\dagger}$ & $1.5^{\dagger}$ & 6.3 \\
\hline & 201722 & 4.05 & 4.21 & 4.25 & 2.0 & 3.1 & 3.0 & -7.0 & -1.8 & -1.0 & $8.8^{*}$ & $7.1^{*}$ & $7.7^{*}$ & $0.5^{\dagger}$ & $2^{+}$ & $2.4^{\dagger}$ \\
\hline & 201723 & 9.31 & 9.49 & 9.77 & 2.2 & 3.0 & 1.4 & -5.1 & -2.4 & -0.4 & $2.5^{* \dagger}$ & $2.6^{* \dagger}$ & $6.9^{*}$ & $1.3^{\dagger}$ & $1.8^{\dagger}$ & 5.5 \\
\hline & 201724 & 15.39 & 15.62 & 16.12 & 1.7 & 3.6 & 1.8 & -4.0 & -2.5 & -0.6 & 3 & $1.8^{\dagger}$ & 4.8 & $2.4^{\dagger}$ & $1.5^{\dagger}$ & 4.3 \\
\hline & 201725 & 11.11 & 11.28 & 11.76 & 2.4 & 3.3 & 1.9 & -6.1 & -4.2 & -0.9 & $1.2^{\dagger}$ & $1.5^{\dagger}$ & 4.2 & $0.8^{\dagger}$ & $1.2^{\dagger}$ & 3.7 \\
\hline \multirow[t]{5}{*}{$\mathbf{K}$} & 201721 & 6.3 & 6.4 & 6.3 & 1.2 & 0.6 & 0.8 & 0.6 & 0.6 & 0.4 & $6.4^{*}$ & $12.8^{*}$ & $9.2^{*}$ & 4.7 & 9.6 & 6.9 \\
\hline & 201722 & 2.7 & 2.8 & 2.7 & 1.0 & 1.2 & 0.9 & -2.3 & -0.5 & -2.3 & $16.6^{*}$ & $14.7^{*}$ & $17.3^{*}$ & 3.8 & 4.7 & 4 \\
\hline & 201723 & 4.3 & 4.4 & 4.3 & 0.5 & 0.6 & 0.8 & 0.4 & 0.1 & 0.5 & $21.8^{*}$ & $20.0^{*}$ & $14.4^{*}$ & 10.9 & 10.5 & 7.2 \\
\hline & 201724 & 6.0 & 6.0 & 6.0 & 0.5 & 0.5 & 0.9 & 1.0 & 0.7 & 0.6 & $14.5^{*}$ & $17.0^{*}$ & $8.6^{*}$ & 9.8 & 12 & 5.9 \\
\hline & 201725 & 4.7 & 4.8 & 4.7 & 0.7 & 0.3 & 1.1 & -1.0 & -1.1 & -1.2 & $14.1^{*}$ & $31.1^{*}$ & $8.7^{*}$ & 7.3 & 16.4 & 4.4 \\
\hline \multirow[t]{5}{*}{$\mathrm{Na}$} & 201721 & 157 & 159 & 155 & 0.8 & 0.6 & 0.7 & 0.1 & 0.4 & -1.4 & $3.2^{*}$ & $3.6^{*}$ & $1.8^{*+}$ & 5.1 & 6.1 & 3.9 \\
\hline & 201722 & 108 & 111 & 112 & 0.8 & 0.9 & 0.8 & -2.5 & 0.3 & 1.0 & $1.4^{* \dagger}$ & $3.6^{*}$ & $3.3^{*}$ & $1.8^{\dagger}$ & 4 & 3.9 \\
\hline & 201723 & 128 & 130 & 129 & 0.4 & 0.8 & 0.8 & -0.8 & 0.5 & 0.3 & $5.2^{*}$ & $3.4^{*}$ & $3.5^{*}$ & 7.1 & 4.5 & 4.6 \\
\hline & 201724 & 153 & 154 & 153 & 0.5 & 0.5 & 1.1 & 0.3 & 2.2 & 0.5 & $5.0^{*}$ & $0.9^{* \dagger}$ & $2.0^{*+}$ & 8.1 & 3.8 & 3.3 \\
\hline & 201725 & 133 & 135 & 135 & 0.5 & 0.5 & 1.2 & -2.1 & -0.7 & -1.0 & $1.5^{\dagger}$ & 5.1 & $1.7^{\dagger}$ & 3.5 & 7.4 & $2.7^{\dagger}$ \\
\hline \multirow[t]{5}{*}{ Cl } & 201721 & 108 & 107 & 105 & 0.8 & 0.7 & 0.9 & 0.7 & 1.7 & -2.6 & 5.3 & 5 & $2.7^{\dagger}$ & 4.1 & 3.5 & $1.6^{\dagger}$ \\
\hline & 201722 & 76 & 72 & 72 & 0.6 & 0.8 & 1.2 & 0.9 & 3.9 & -1.4 & 7.2 & $1.4^{\dagger}$ & 3 & 5.5 & $0.1^{\dagger}$ & $2.1^{\dagger}$ \\
\hline & 201723 & 89 & 86 & 85 & 0.4 & 0.6 & 0.9 & 0.7 & 2.9 & -2.0 & 10 & 3.7 & 3.3 & 7.7 & $2^{\dagger}$ & $2.2^{\dagger}$ \\
\hline & 201724 & 108 & 106 & 105 & 0.6 & 0.5 & 0.6 & 0.8 & 2.1 & -0.9 & 6.9 & 5.6 & 6.6 & 5.3 & 3.7 & 5 \\
\hline & 201725 & 96 & 94 & 93 & 0.5 & 0.4 & 0.7 & -1.1 & 1.4 & -2.8 & 7.7 & 9.7 & 3 & 5.7 & 7 & 1.6 \\
\hline \multirow[t]{5}{*}{$\mathrm{Ca}$} & 201721 & 3.14 & 3.21 & 3.03 & 1.2 & 1.0 & 1.9 & 1.2 & 3.5 & -1.0 & $5.6^{*}$ & $4.4^{*}$ & $3.8^{*}$ & 3.1 & $1.5^{\dagger}$ & $2.1^{\dagger}$ \\
\hline & 201722 & 1.84 & 1.92 & 1.80 & 1.2 & 0.8 & 1.7 & 1.2 & 4.4 & -1.3 & $10.5^{*}$ & $10.9^{*}$ & $7.4^{*}$ & 3.3 & $0.8^{\dagger}$ & $2.2^{+}$ \\
\hline & 201723 & 2.73 & 2.82 & 2.67 & 0.8 & 0.8 & 1.3 & 1.1 & 3.8 & -1.5 & $10.4^{*}$ & $6.5^{*}$ & $5.9^{*}$ & 5 & $1.5^{\dagger}$ & $2.7^{\dagger}$ \\
\hline & 201724 & 3.02 & 3.12 & 2.92 & 0.8 & 0.7 & 1.2 & 0.8 & 4.0 & -2.0 & $9.1^{*}$ & $6.0^{*}$ & $5.3^{*}$ & 5.1 & $1.4^{\dagger}$ & $2.5^{\dagger}$ \\
\hline & 201725 & 2.72 & 2.81 & 2.65 & 0.6 & 0.7 & 0.9 & 0.0 & 2.1 & -3.4 & $14.7^{*}$ & $9.9^{*}$ & $6.5^{*}$ & 8.8 & 4.2 & $1.7^{\dagger}$ \\
\hline
\end{tabular}

*TEa calculated by using absolute bias to calculate $\sigma$; not marked indicates using percentage bias to calculate $\sigma$. ${ }^{\dagger}$ Sigma values $<3$. $\mathrm{TEa}_{\mathrm{CLIA}}$ - allowable total error derived from US Clinical Laboratory Improvement Amendments 1988 (CLIA '88). TEa WS/T $^{-}$allowable total error derived from the People's Republic of China Health Industry Standard (WS/T403-2012). Alb - albumin, g/L. ALT - alanine aminotransferase, $\mathrm{U} / \mathrm{L} 37^{\circ} \mathrm{C}$. BT - bilirubin, total, $\mu \mathrm{mol} / \mathrm{L}$. Glc - glucose, mmol/L. CREA - creatinine, $\mu \mathrm{mol} / \mathrm{L}$. Urea, $\mathrm{mmol} / \mathrm{L}$. K potassium, mmol/L. $\mathrm{Na}$ - sodium, $\mathrm{mmol} / \mathrm{L}$. Cl - chlorides, mmol/L. Ca - calcium, mmol/L. 
and with different analysers are shown in Supplementary Figure 1.

\section{IQC Sigma metrics}

The $\sigma$ metrics for the ten assays calculated from internal QC data (June 2017) are shown in Table 4. Similar to the PT results, $\sigma_{\mathrm{WS} / \mathrm{T}}$ was $<\sigma_{\mathrm{CLIA}}$ in 9 out of 10 assays (all except $\mathrm{Na}$ ), with ALT, BT and Glc reaching $6 \sigma$; Na reaching $4 \sigma$; and $\mathrm{K}$ and $\mathrm{Ca}$ reaching $3 \sigma$ on the Beckman AU5800 analyser. On the Roche $\mathrm{C} 8000$ analyser, GIc, CREA, K and Na reached the $6 \sigma$ level; ALT, BT and Urea reached $4 \sigma$; and $C a$ reached $3 \sigma$. For the Siemens Dimension analyser, CREA reached $6 \sigma$, Alb reached $5 \sigma$, and Glc and K reached $3 \sigma$.

For $\sigma_{\mathrm{CLIA}}$, all 10 assays were above $3 \sigma$ on the Beckman AU5800 analyser, of which ALT, BT, CREA and Ca achieved $6 \sigma$ at two levels of QC materials. For the Roche $\mathrm{C} 8000$ system, $\sigma_{\mathrm{CLIA}}<1 \sigma$ for $\mathrm{Cl}$, whereas all other nine items were $>3 \sigma$, and $B T, C R E A, K$ and Ca achieved $6 \sigma$ while ALT reached $5 \sigma$. For the Siemens Dimension analyser, the $\sigma_{\text {CLIA }}$ value for Glc, $\mathrm{Na}$ and $\mathrm{Cl}$ was $<3$, but Alb, CREA, $\mathrm{K}$ and $\mathrm{Ca}$ achieved $6 \sigma$. Supplementary Figure 2 displays more intuitive $\sigma_{\mathrm{CLIA}}$ levels for the 10 assays at various concentrations using the three analysers. We also calculated $\sigma$ metrics for the 10 assays based on IQC data from January - June 2017 and observed some differences compared with the $\sigma$ metrics calculated from the IQC data in June (Supplementary Table 1).

\section{Comparison of $\sigma$ Metrics Between Methods}

In Figure 1, the differences in $\sigma_{\mathrm{CLIA}}$ as calculated using the two methods and three analysers are shown. For some analytes, the values of $\sigma_{\mathrm{CLIA}}$ derived from the two approaches are significantly different. For example, $\sigma_{\mathrm{CLIA}}$ for the PT-based approach versus the IQC-based approach at similar concentrations was 6.5 (201722) versus 3.9 (45751) for Alb and 1.4 (201722) versus 4.6 (45751) for Na on the Beckman AU5800 analyser. To allow comparison of the differences between the two methods of calculating $\sigma_{C L I A}$ at similar concentrations, we have listed the $\sigma_{\mathrm{CLIA}}$ values from IQC materials and PT samples in Supplementary Figure 3.

\section{Discussion}

Evaluating the quality of laboratory testing is an important research topic in clinical laboratories. Six Sigma quality standards take bias (system error) and CV (random error) into account to systematically and extensively guide quality management in clinical laboratories while analysing possible causes of error, identifying solutions, better assuring testing quality and optimizing the QC schedule. However, the optimum TEa, bias, CV, and other indicators to calculate $6 \sigma$ remain unclear, particularly when the sources of bias and CV vary between laboratories. We therefore compared two new approaches to calculate $\sigma$ metrics as a future reference for the application of $6 \sigma$ quality management in clinical laboratories.

To our knowledge, the present study is the first to use PT samples to assess imprecision and further calculate $\sigma$ values. We obtained CV values from these samples to ensure that the same source of bias and CV were used in $\sigma$ calculations. Given that PT samples typically have five different levels of concentrations, and it is easier to cover different levels of an assay for medical decision-making, this approach conveniently evaluates $\sigma$ at different concentrations to better indicate analyser performance. A limitation of the PT-based approach is the assessment of short-term imprecision, which may lead to a lower CV and overestimated $\sigma$ value. According to the manufacturer's instructions for the PT materials, prepared samples are stable for only seven days, and so are unsuitable for use in long-term evaluations.

To compare differences in $\sigma$ levels calculated by the two methods, we used the relatively long-term CV calculated in the same month of IQC data in the IQC-based approach, thus accounting for other factors (batch number, instrument status, calibration, personnel, temperature, humidity, etc.). Our findings indicate that, for some assays, the $\sigma$ values derived from the two approaches are significantly different. These differences may clearly have significant outcomes for QC rule selection; for example, a $4 \sigma$ method requires multi-rule QC while a $6 \sigma$ method can be controlled by a simple, single-rule QC. 
There may be several reasons for the difference between the two approaches. First, there may be obvious differences in analyte concentrations be-
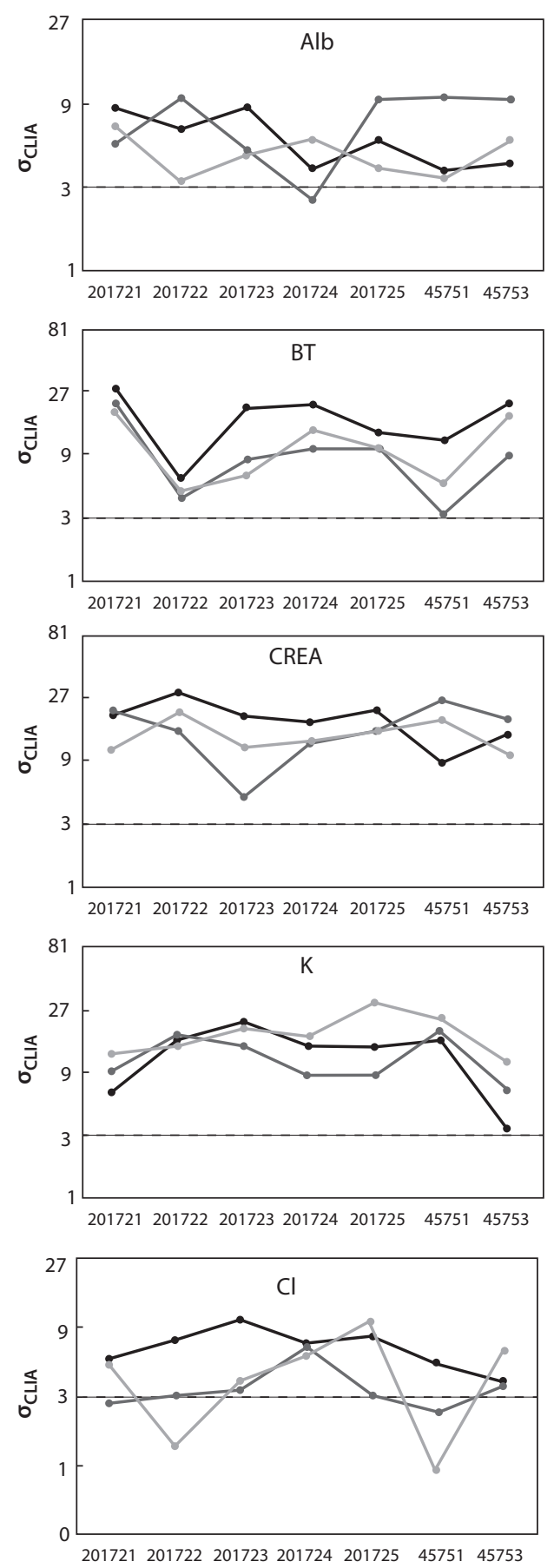

tween the IQC control samples and the PT materials. Second, the group mean might not have been appropriate. Third, systematic deviations may have
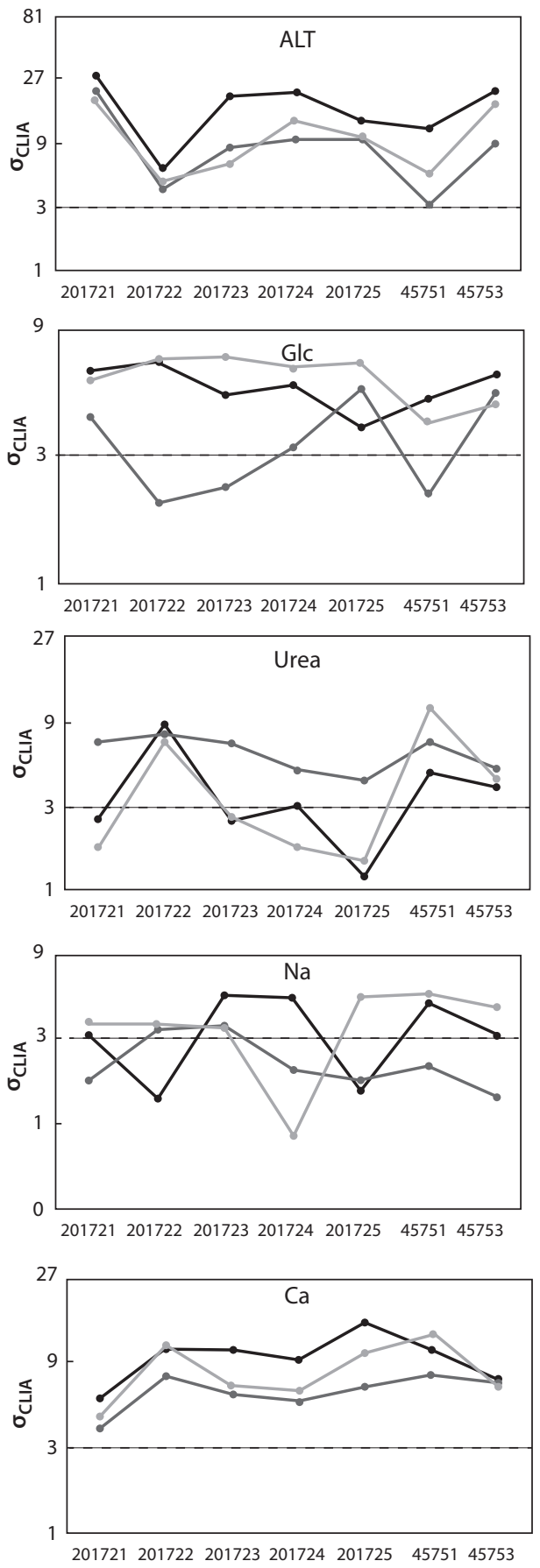

Beckman AU5800

Roche

Siemens Dimension

Figure 1. Comparison of $\sigma_{\text {CLIA }}$ values calculated using two methods for the same test item. Note: 201721-201725 represent the lot number of proficiency testing materials, and 45751 and 45752 represent the lot number of Bio-Rad chemistry quality control materials. The horizontal lines indicate the $3 \sigma$ level line. Alb - albumin, g/L. ALT - alanine aminotransferase, U/L $37^{\circ} \mathrm{C}$. BT - bilirubin, total, $\mu \mathrm{mol} / \mathrm{L}$. Glc - glucose, mmol/L. CREA - creatinine, $\mu \mathrm{mol} / \mathrm{L}$. Urea, mmol/L. K - potassium, mmol/L. $\mathrm{Na}$ - sodium, $\mathrm{mmol} / \mathrm{L}$. Cl - chlorides, $\mathrm{mmol} / \mathrm{L}$. Ca - calcium, $\mathrm{mmol} / \mathrm{L}$. 
been present, possibly due to the short detection time period when evaluating $\sigma$ with PT. In addition, the $\sigma$ value itself is influenced by both bias and imprecision, and in clinical laboratories, multiple factors can influence these parameters. Where the $\sigma$ value is not satisfactory, the cause should be determined, whether it is a bias or an imprecision issue, and an appropriate solution should be identified. In the PT-based approach, the imprecision was mostly acceptable, and the suboptimal $6 \sigma$ values may have been mainly attributable to bias. If a laboratory wants to use individualized quality con- trol rules, it may select one method for evaluating $\sigma$ level and use another method for verification.

We also compared the $\sigma$ calculation in the IQCbased approach during different time periods and found that the 1- and 6-month values differed significantly for some analytes (Table 4 and Supplementary Table 1). As greater imprecision is expected with prolonged time, 6-month $\sigma$ values were expected to be lower. However, this was not observed in all cases; for example, Glc (low QC level, 45751) on the Siemens analyser was 2.5 at 1 month versus 5.1 at 6 months, and Urea (low QC level,

TABLE 4. Sigma metrics for the IQC-based approach using one-month IQC data

\begin{tabular}{|c|c|c|c|c|c|c|c|c|c|c|c|c|c|c|c|c|}
\hline \multirow[b]{2}{*}{ Item } & \multirow[b]{2}{*}{$\begin{array}{c}\text { Control } \\
\text { level }\end{array}$} & \multicolumn{3}{|c|}{ Mean } & \multicolumn{3}{|c|}{ CV (\%) } & \multicolumn{3}{|c|}{ Bias (\%) } & \multicolumn{3}{|c|}{$\sigma_{\mathrm{CLIA}}$} & \multicolumn{3}{|c|}{$\sigma_{W S / T}$} \\
\hline & & 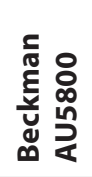 & 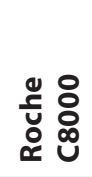 & 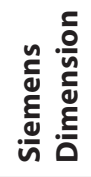 & 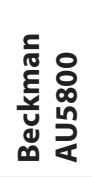 & 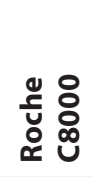 & 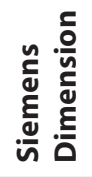 & 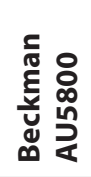 & 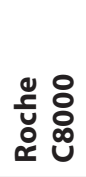 & 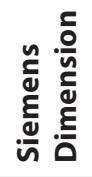 & 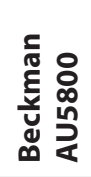 & 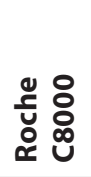 & 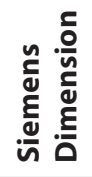 & 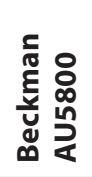 & $\begin{array}{l}\text { 용 웅 } \\
\text { 용 }\end{array}$ & 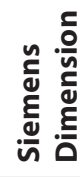 \\
\hline \multirow[t]{2}{*}{ Alb } & L & 24 & 26 & 24 & 2.0 & 2.7 & 1.0 & -2.7 & -1.1 & -0.2 & 3.7 & 3.3 & 9.8 & $1.7^{\dagger}$ & $1.8^{\dagger}$ & 5.8 \\
\hline & $\mathrm{H}$ & 39 & 42 & 40 & 1.9 & 1.4 & 1.0 & -2.3 & -2.3 & 0.6 & 4.1 & 5.5 & 9.4 & $2^{+}$ & $2.6^{+}$ & 5.4 \\
\hline \multirow[t]{2}{*}{ ALT } & $\mathrm{L}$ & 31 & 29 & 37 & 1.7 & 3.1 & 2.7 & 0.8 & 3.4 & 11.5 & 10.8 & 5.4 & 3.1 & 8.5 & 4.1 & $1.6^{\dagger}$ \\
\hline & $\mathrm{H}$ & 184 & 173 & 200 & 0.9 & 1.1 & 1.4 & 0.7 & 1.5 & 8.2 & 17.2 & 16.9 & 8.6 & 12.8 & 13.2 & 5.7 \\
\hline \multirow[t]{2}{*}{ BT } & $\mathrm{L}$ & 10.1 & 8.8 & 11.0 & 1.6 & 2.5 & 2.8 & 1.5 & -4.4 & 13.0 & $41.8^{*}$ & $29.3^{*}$ & $18.0^{*}$ & 8.5 & 4.2 & $0.7^{\dagger}$ \\
\hline & $\mathrm{H}$ & 115.4 & 108.2 & 116.6 & 1.3 & 0.9 & 0.7 & 0.6 & -2.6 & -1.2 & 15 & 19.3 & 26.9 & 11.1 & 13.7 & 19.7 \\
\hline \multirow[t]{2}{*}{ GIc } & L & 3.3 & 3.3 & 3.5 & 1.2 & 1.2 & 2.4 & -1.2 & -2.2 & 1.7 & 4.8 & 4 & $2.2^{\dagger}$ & 7.3 & 6.5 & 3.5 \\
\hline & $\mathrm{H}$ & 20.3 & 20.1 & 20.8 & 1.1 & 1.1 & 1.2 & -0.5 & -1.9 & 0.8 & 5.9 & 4.6 & 5.1 & 8.6 & 7.4 & 7.6 \\
\hline \multirow[t]{2}{*}{ CREA } & L & 78 & 81 & 75 & 3.3 & 1.7 & 1.4 & 5.3 & 1.3 & 0.6 & $8.5^{*}$ & $18.1^{*}$ & $24.8^{*}$ & $1.8^{\dagger}$ & 6.3 & 8.1 \\
\hline & $\mathrm{H}$ & 848 & 821 & 809 & 0.9 & 1.5 & 0.8 & 2.0 & -0.5 & -0.2 & 13.9 & 9.7 & 18.5 & 10.6 & 7.7 & 14.8 \\
\hline \multirow[t]{2}{*}{ Urea } & L & 5.05 & 4.9 & 5.24 & 3.1 & 1.7 & 2.7 & -4.8 & 0.1 & -0.3 & $4.7^{*}$ & $10.1^{*}$ & $7.1^{*}$ & $1^{\dagger}$ & 4.7 & $2.9^{\dagger}$ \\
\hline & $\mathrm{H}$ & 27.15 & 26.3 & 28.17 & 2.1 & 2.1 & 1.8 & -0.8 & -0.1 & -0.3 & 3.9 & 4.3 & 4.8 & 3.4 & 3.8 & 4.3 \\
\hline \multirow[t]{2}{*}{$\mathbf{K}$} & L & 2.6 & 2.6 & 2.4 & 1.2 & 0.8 & 1.0 & 0.7 & -0.8 & -2.2 & $16.1^{*}$ & $24.0^{*}$ & $18.6^{*}$ & 4.4 & 6.5 & 3.8 \\
\hline & $\mathrm{H}$ & 8.0 & 8.1 & 7.9 & 1.5 & 0.6 & 0.7 & 1.5 & -0.1 & -1.5 & $3.3^{*}$ & $10.8^{*}$ & $6.6^{*}$ & 3 & 9.9 & 6.5 \\
\hline \multirow[t]{2}{*}{$\mathrm{Na}$} & L & 115 & 115 & 115 & 0.8 & 0.6 & 0.8 & 0.0 & -0.4 & -1.7 & $4.6^{*}$ & $5.2^{*}$ & $2.1^{* \dagger}$ & 5 & 6 & $2.9^{\dagger}$ \\
\hline & $\mathrm{H}$ & 158 & 161 & 157 & 0.8 & 0.5 & 0.6 & -0.2 & -0.2 & -1.6 & $3.1^{*}$ & $4.4^{*}$ & $1.4^{* \dagger}$ & 4.8 & 7.5 & 3.9 \\
\hline \multirow[t]{2}{*}{$\mathrm{Cl}$} & $\mathrm{L}$ & 78 & 76 & 75 & 0.8 & 0.7 & 0.8 & 0.9 & 4.4 & -3.2 & 5.1 & $0.9^{\dagger}$ & $2.3^{\dagger}$ & 3.8 & $-0.6^{\dagger}$ & $1^{\dagger}$ \\
\hline & $\mathrm{H}$ & 118 & 117 & 119 & 1.1 & 0.6 & 0.5 & 0.8 & 1.3 & -3.3 & 3.8 & 6.2 & 3.5 & $2.9^{\dagger}$ & 4.5 & $1.5^{\dagger}$ \\
\hline \multirow[t]{2}{*}{$\mathrm{Ca}$} & L & 1.48 & 1.51 & 1.4 & 1.6 & 1.2 & 1.9 & 0.1 & 0.7 & -3.2 & $10.4^{*}$ & $12.6^{*}$ & $7.5^{*}$ & 3.1 & 3.6 & $0.9^{\dagger}$ \\
\hline & $\mathrm{H}$ & 3.28 & 3.35 & 3.22 & 0.9 & 0.9 & 1.1 & 0.9 & 1.6 & 0.6 & $7.1^{*}$ & $6.6^{*}$ & $6.8^{*}$ & 4.5 & 3.8 & 4.2 \\
\hline
\end{tabular}

*Allowable total error (TEa) calculated by using absolute bias to calculate $\sigma$; not marked indicates using percentage bias to calculate $\sigma .{ }^{\dagger}$ Sigma values < 3. TEa $\mathrm{CLIA}$ - allowable total error derived from US Clinical Laboratory Improvement Amendments 1988 (CLIA '88). $\mathrm{TEa}_{\mathrm{WS} / \mathrm{T}}$ - allowable total error derived from the People's Republic of China Health Industry Standard (WS/T403-2012). Alb - albumin, $\mathrm{g} / \mathrm{L}$. ALT - alanine aminotransferase, U/L $37^{\circ} \mathrm{C}$. BT - bilirubin, total, $\mu \mathrm{mol} / \mathrm{L}$. Glc - glucose, mmol/L. CREA - creatinine, $\mu \mathrm{mol} / \mathrm{L}$. Urea, $\mathrm{mmol} / \mathrm{L}$. K - potassium, $\mathrm{mmol} / \mathrm{L}$. $\mathrm{Na}$ - sodium, $\mathrm{mmol} / \mathrm{L}$. Cl - chlorides, mmol/L. Ca - calcium, mmol/L. 
45751) on the Beckman analyser was 4.7 versus 7.2. From the original data analysis, the difference in Glc on the Siemens analyser was due to the larger CV observed in June. The difference in Urea on the Beckman analyser, however, may have been the result of periodic biases with different signs averaged to an ignorable long-term bias. In practice, laboratories must take caution when implementing "Westgard Sigma Rules" in quality control based on $\sigma$ values, because these values may change continuously with respect to precision and bias arising, for example, from calibrations, reagents, or personnel (4). As described previously, $\sigma$ value between different periods for some analytes may differ, laboratory should monitor the $\sigma$ level continuously when using individualized quality control rules based on the $\sigma$ evaluation.

Bias can significantly impact the $\sigma$ metric. It can (theoretically) be corrected, while imprecision is more difficult to influence. However, bias is generally more difficult to estimate. The most reliable way to do so is to use a reference method. Most studies, including the present study, use group means from EQA data on the same instruments and methods as a target, rather than the reference method. Therefore, the observed bias is only "arbitrary" instead of "true". External quality assessment peer group evaluation has also been shown to be insufficient in determining analytical quality and may compromise patient care, despite its acceptance by participating laboratories and manufacturers (14). This approach is therefore a limitation of the present study, but also represents a common limitation of $6 \sigma$ for quality management at present, as most routine laboratory testing does not have a reference method that can be conveniently implemented. Determining bias from proficiency testing or global QC reports thus remains the primary approach in current $6 \sigma$ evaluations. It is therefore critical to select the appropriate group when using group mean to assess bias. In the PTbased approach used in our study, group means were calculated after excluding data more than two standard deviations away from the mean in order to exclude extreme values. Compared with the IQC-based approach, the relatively small number of laboratories using Siemens instruments $(<$
10) in the PT-based approach may have affected the reliability of the means. This limitation should be considered when selecting an appropriate method for $\sigma$ calculation.

The TEa is another important parameter in $\sigma$ calculations, and extensive efforts to understand, establish and unify the quality of testing and analysis are ongoing. In May 2014, the European Federation of Clinical Chemistry and Laboratory Medicine (EFLM) held its first meeting on countermeasures in Milan, Italy, under the theme "Analytical performance targets set 15 years after the Stockholm Conference". At the conference, experts made in-depth and detailed discussions on the progress and further understanding of setting up analytical performance goals in clinical laboratories in the 15 years after the Stockholm meeting, and also issued a statement of synergies after the meeting (15). At present, TEa values are primarily derived from CLIA guidelines, although few reports have used biological variations $(6,7,9,16,17)$. In China, the Ministry of Health published analytical quality specifications for routine clinical biochemistry (WS/T 403-2012) in 2012, derived from data on within-subject and between-subject biologic variation, while taking into account the quality of analysis currently achievable. However, these standards are expert based and have the objective (at least for CLIA) to set broad quality limits that will include the majority of laboratories; CLIA guidelines, for example, are often considered "loose" in terms of analytical performance. The TEa value selection can lead to significant differences in the evaluation of $\sigma$ values (10). This limitation should be considered when selecting an appropriate TEa for $\sigma$ calculation.

We also compared the effects of TEa values on $\sigma$ calculations. The $\sigma_{\mathrm{WS} / \mathrm{T}}$ value was significantly lower than that of $\sigma_{\mathrm{CLIA}}$ in most assays, given that $\mathrm{TEa}_{\mathrm{WS} / \mathrm{T}}$ is more stringent than $\mathrm{TEa}_{\mathrm{CLIA}}$. For some assays, the analyser could not achieve even the $3 \sigma$ level. In addition, absolute bias was used for the $\mathrm{K}$, $\mathrm{Na}$ and $\mathrm{Ca}$ assays in the CLIA guidelines, and BT, Glc, Urea and CREA at the low levels, but all percentage bias was used for $\mathrm{TEa}_{\mathrm{WS} / \mathrm{T}}$. Therefore, for low-concentration specimens (201722, 45751), $\sigma_{\text {CLIA }}$ was significantly higher than $\sigma_{W S / T}$ for BT, 
CREA, Urea, $\mathrm{K}$ and $\mathrm{Ca}$. When screening TEa sources, the source most closely related to the performance for a given laboratory should be selected to ensure continuous improvement in quality management. Laboratories should not pursue the best $\sigma$ metrics as a laboratory goal, nor should they select the most stringent TEa sources, to avoid unnecessary burden on laboratories.

In the present study, only the reagents for CREA and BT on the Beckman AU5800 system were not obtained from the original manufacturer. The results showed a minimum $\sigma_{\mathrm{CLIA}}$ for BT and CREA of 5.6 and 17.4, respectively, when calculated from proficiency testing samples, and 7.4 and 8.4, respectively, when calculated from IQC. Both results were satisfactory. These findings indicate that both domestic and foreign reagents selected for routine laboratory testing can achieve a high quality level.

The performance of the analysers was also compared. The Beckman AU5800 and Roche C8000 systems each reached $3 \sigma$ levels for seven assays, while the Siemens Dimension analyser received $3 \sigma$ levels for five assays. Different assays showed variations in performance among the analysers, although these variations were not significantly different. A laboratory may select an analyser based on assay usage frequency while still considering the $\sigma$ evaluation, thereby personalizing the selec- tion. Different assays may be also assigned to different instruments based on these results. We found that, for the Siemens Dimension system, $\sigma$ assessed by both methods had multiple values $<3$ (14\% for PT samples, 20\% for IQC materials). Given that the Siemens Dimension instrument in our laboratory has been in daily use for more than 5 years, it was replaced by a new instrument in December 2017.

In conclusion, both methods of evaluating $\sigma$ in this study can be used to assess the performance of a specific analyser, despite the observed differences in $\sigma$ calculated by different methods. In the practical application of $\sigma$ metrics for QC management, $\sigma$ should be evaluated multiple times when optimizing a QC schedule.

\section{Acknowledgments}

This study was financially supported by the China Capital Health Research and Development of Special Project (2016-2-4017), the National Key Technology Research and Development Support program (2015BAI32H00), the Beijing Municipal Science\& Technology Commission (Z161100001816043), and research grants from the National Natural Science Foundation of China (81702060).

\section{Potential conflict of interest}

None declared.

\section{References}

1. Forsman RW. Why is the laboratory an afterthought for managed care organizations? Clin Chem 1996;42:813-6.

2. Nevalainen D, Berte L, Kraft C, Leigh E, Picaso L, Morgan $T$. Evaluating laboratory performance on quality indicators with the six sigma scale. Arch Pathol Lab Med 2000;124:516-9.

3. Westgard JO, Westgard SA. Assessing quality on the Sigma scale from proficiency testing and external quality assessment surveys. Clin Chem Lab Med 2015;53:1531-5. https:// doi.org/10.1515/cclm-2014-1241

4. Westgard JO, Bayat $H$, Westgard SA. Planning risk-based SQC schedules for bracketed operation of continuous pro-

duction analysers. Clin Chem 2018;64:289-96. https://doi. org/10.1373/clinchem.2017.278291

5. Westgard JO, Westgard SA. Quality control review: implementing a scientifically based quality control system. Ann Clin Biochem 2016;53:32-50. https://doi. org/10.1177/0004563215597248

6. Shaikh MS, Moiz B. Analytical performance evaluation of a high-volume hematology laboratory utilizing sigma metrics as standard of excellence. Int J Lab Hematol 2016;38:193-7. https://doi.org/10.1111/ijlh.12468

7. Singh B, Goswami B, Gupta VK, Chawla R, Mallika V. Application of sigma metrics for the assessment of quality assu- 
rance in clinical biochemistry laboratory in India: a pilot study. Indian J Clin Biochem 2011;26:131-5. https://doi. org/10.1007/s12291-010-0083-1

8. Tran MT, Hoang K, Greaves RF. Practical application of biological variation and Sigma metrics quality models to evaluate 20 chemistry analytes on the Beckman Coulter AU680. Clin Biochem 2016;49:1259-66. https://doi.org/10.1016/j. clinbiochem.2016.08.008

9. Nanda SK, Ray L. Quantitative application of sigma metrics in medical biochemistry. J Clin Diagn Res 2013;7:2689-91. https://doi.org/10.7860/JCDR/2013/7292.3700

10. Hens K, Berth M, Armbruster D, Westgard S. Sigma metrics used to assess analytical quality of clinical chemistry assays: importance of the allowable total error (TEa) target. Clin Chem Lab Med 2014;52:973-80. https://doi.org/10.1515/ cclm-2013-1090

11. Clinical and Laboratory Standard Institute (CLSI). User Verification of Precision and Estimation of Bias: Approved Guideline. EP15A3, 3rd ed. Wayne: CLSI, 2014.

12. CLIA Requirements for Analytical Quality. Available at: https://www.westgard.com/clia.htm/. Accessed February 5th, 2017.
13. People's Republic of China Health Industry Standard (WS/ T403-2012), 2012. Available at: http://www.clinet.com.cn/ sigmapv/. Accessed November 20th 2017.

14. Friedecky B, Kratochvila J, Budina M. Why do different EQA schemes have apparently different limits of acceptability? Clin Chem Lab Med 2011;49:743-5. https://doi.org/10.1515/ CCLM.2011.105

15. Sandberg S, Fraser CG, Horvath AR, Jansen R, Jones G, Oosterhuis $W$, et al. Defining analytical performance specifications: consensus statement from the 1st Strategic Conference of the European Federation of Clinical Chemistry and Laboratory Medicine. Clin Chem Lab Med 2015;53:833-5. https://doi.org/10.1515/cclm-2015-0067

16. Huysal K, Budak YU. Application of sigma metrics for the assessment of quality assurance using the MQ-2000 PT HbA1c analyzer. Biochem Med (Zagreb) 2015;25:416-20. https://doi.org/10.11613/BM.2015.042

17. Ricos C, Alvarez V, Cava F, Garcia-Lario JV, Hernandez A, Jimenez $C V$, et al. Current databases on biological variation: pros, cons and progress. Scand J Clin Lab Invest 1999;59:491500. https://doi.org/10.1080/00365519950185229 